\title{
Dermatoscopy Using Multi-Layer Perceptron, Convolution Neural Network, and Capsule Network to Differentiate Malignant Melanoma From Benign Nevus
}

Shamik Tiwari, Department of Virtualization, School of Computer Science, University of Petroleum and Energy Studies, Dehradun, India

\begin{abstract}
Epiluminescence microscopy, more simply, dermatoscopy, entails a process using imaging to examine skin lesions. Various sorts of skin ailments, for example, melanoma, may be differentiated via these skin images. With the adverse possibilities of malignant melanoma causing death, an early diagnosis of melanoma can impact on the survival, length, and quality of life of the affected victim. Image recognition-based detection of different tissue classes is significant to implementing computeraided diagnosis via histological images. Conventional image recognition require handcrafted feature extraction before the application of machine learning. Today, deep learning is offering significant choices with the progression of artificial learning to defeat the complications of the handcrafted feature extraction methods. A deep learning-based approach for the recognition of melanoma via the Capsule network is proposed here. The novel approach is compared with a multi-layer perceptron and convolution network with the Capsule network model yielding the classification accuracy at $98.9 \%$.
\end{abstract}

\section{KEYWORDS}

Capsule Network, Convolution Neural Network, Dermatoscopy, Multi-Layer Perceptron

\section{INTRODUCTION}

Malignant melanoma (or, melanoma) is a form of human skin cancer arising from the melanocytes (pigment-containing cells). The affected cells are usually found in the human skin, but rarely in the mouth, eye or intestines. Normally appearing on the legs of women and backs of the men, the affected cells can also grow from a mole with some changes such as an escalation in size, uneven edges, color change, irritation, and skin breakdown. With the adverse possibilities of malignant melanoma causing death, an early diagnosis of melanoma can aid the affected victim to survive and live longer.

Although strange to behold, a mole, also referred to as dysplastic nevi, is typically benign (noncancerous). Moles may have an appearance similar to melanoma, and a person who has them is at a higher risk of harboring melanoma in a mole or somewhere else on the skin. The larger the number of moles one has, the greater the risk, for instance, someone who has ten (10) or more moles will 
have about twelve (12) times the threat of having melanoma v. someone who only has a single or no mole at all (Argenziano et al., 2012).

In dermatology, melanoma is identified via clinical screening through the use of a device known as digital epiluminescence dermatoscope (or, dermatoscope) by a trained dermatologist. The skin lesion image is typically analyzed by the ABCD rule where A refers to asymmetry, B to border irregularities, $\mathrm{C}$ to color patterns and D to diameter (Rigel et al., 2010). A 7-point checklist, encompassing: (1) strange pigment network; (2) gray-blue areas; (3) strange vascular pattern; (4) radial streaming; (5) uneven diffuse pigmentation; (6) globules, uneven dots; and (7) regression pattern is also used to assist dermatologists in this task (Argenziano et al., 1998). Further biopsy tests are often ordered should the initial screening of the patient indicate any symptoms of melanoma. As the use of conventional approaches is often costly and time-consuming with biopsy tests often having several side effects, nowadays, image analysis and machine learning based systems are highly recommended for melanoma detection via dermoscopic images (Singhal et al., 2015; Ker et al., 2018). Moreover, the limited availability of trained dermatologists has deterred the use of conventional melanoma detection methods.

The rest of the work is organized as follows. The next section (Section 2) overviews the extant literature. Section 3 presents three different machine learning classification models for identifying malignant (cancerous) lesions. Section 4 details the experiment, comprising image dataset description, performance measures, and simulation results. The final section (Section 5) provides concluding remarks relating to the key contributions, limitations, practical implications and potential future work.

\section{LITERATURE REVIEW}

Computer-assisted melanoma detection from dermoscopy images is more efficient than manual approach though it a very challenging task. A key challenge here is the enormous intra-class similarities of melanomas and nevus. Melanoma and nevus share the visual similarities in terms of surface texture, size, color, shape, and position in the dermatoscopy images. Conversely, the comparatively poor contrasts and vague boundaries among skin lesions and usual skin sections may further challenge the computer-assisted identification job. Sometimes, the existence of image degradation factors, such as the typical veins and hair or sometimes artificially imposed images due to noise or blur degrades the quality of skin lesions, thereby making the identification task harsher (Yu et al., 2017).

To date, researchers have instituted clever ways to resolve this challenging task. Alcon et al. (2009) have proposed a decision support system for melanoma classification. This work has combined the result of the image classification with some context information such as age, gender, skin type and suffered body portion. The system effectively categorized melanoma images with a precision of $86 \%$. More recently, Cavalcanti \& Scharcanski (2011) present an even more innovative system, which comprises image pre-processing, region segmentation, feature extraction, and lesion classification stages. Moreover, a two-stage classification model has been suggested that reconfirm the lesions labeled as benign to reduce the false negative cases. These authors also test their work with two publicly available datasets and achieve an accuracy of $96.71 \%$.

Sheha et al. (2012) discuss an automatic system, which uses Gray Level Co-occurrence Matrix (GLCM) based features and passes these features in Multilayer Perceptron (MLP) classification model for detection. The MLP classifier is proposed in two different manners during the training and testing steps. This work has shown that texture analysis is a respected scheme for the discernment of melanocytic skin tumors with $100 \%$ accuracy for the training set and $92 \%$ accuracy for the test set. MED-NODE, an expert system in this very domain, has emerged (e.g., Giotis et al., 2015). MEDNODE is purportedly competent in supporting physicians with the diagnostic process for melanoma detection. The proposed system utilizes non-dermoscopic images of lesions from which it identifies the skin lesion areas and calculates features based on the texture and color. Most recently, Mukherjee et al. (2018) have utilized a MLP for melanoma detection. They use nature-inspired metaheuristic 
algorithms to find the optimum amount of neurons in hidden layers of an MLP model. The classification accuracy is found to be over $91 \%$ using gray level run length encoding matrix and GLCM based features.

Lately, the accessibility of high computation power and the increasing size of dataset permit the use of deep learning models. Examples of such models being applied in our case include Convolutional Neural Network (CNN or ConvNet) and Capsule Network (CapsNet). These models have been found to achieve extraordinary performance in a wide range of applications, specifically in the image recognition and classification domains. Importantly, these networks can acquire valuable features directly from the input images by optimizing the learning loss function in contrast to the conventional machine learning models that depend largely on handcrafted feature extraction prior to their applications.

Carcagnì et al. (2018) present a Deep CNN, established on the DenseNet architecture to classify seven types of skin lesions: (i) Melanoma, (ii) Benign keratosis, (iii) Basal cell carcinoma, (iv) Actinic keratosis, (v) Melanocytic nevus, (vi) Dermatofibroma, and (vii) Vascular skin lesion. In the validation phase, their proposed system has achieved an overall accuracy of $89.2 \%$. Nanni et al. (2018) have proposed a CNN-based system with a set of descriptors for melanoma classification. The offered system represents an effective method of boosting the efficiency of trained CNNs by combining multiple CNNs into an ensemble. The simulation results on dermatoscopic images display the accuracy up to $88.6 \%$. Altogether, based on image analysis, and definitely in histopathological image analysis, ConvNet deep learning model has been found to achieve exceptional performance in the medical examination.

The selection of the best performing machine-learning model for a particular application has been an open research challenge. This paper addresses the issue in investigating the performance of three different classification models (i.e., multi-layer perceptron (MLP), CNN and CapsNet) for the categorization of melanoma and nevus skin lesions.

\section{MELANOMA AND NEVUS CLASSIFICATION MODELS}

In this section, the architecture for the three different classification models that are to be evaluated in this work is described.

\subsection{Multi-Layer Perceptron (MLP)}

A MLP is a type of feed-forward artificial neural network (ANN) having multiple layers. MLP utilizes a supervised learning method termed backpropagation for training. A single layer perceptron can learn only linear functions, but an MLP is structured to learn linear v. nonlinear relationships embedded in the data. In the past, no rule exists which can specify clearly how NNs may be trained with more than a single layer. Essentially, the ANN is characterized by having three separate layers: (a) an input layer; (b) a hidden layer; and (c) an output layer. The first layer acts as the input layer, receives the network input. The number of neurons in this layer is always going to be equivalent to the number of input parameters. The output layer is the final layer, where results of the network analysis can be received. Sandwiched between the input-output layers is (are) the hidden layer(s). The number of such hidden layer(s) can vary depending on the function(s) to be supported by the hidden layer(s), which is (are) to encode the input so as to map it onto the output (Tiwari et al., 2011; Kruse et al., 2013). To date, data sciences in the ANN domain have verified that an MLP model with just a single hidden layer can easily approximate or mimic any function to associate its input with its output (Esteves et al., 2018). Figure 1 displays the general architecture of the MLP model.

Overall, the number of neurons in the MLP NN layers is largely dependent on the number of classes in the problem. In the case of the skin lesion classification work to be differentiated via a 3-layer MLP NN (input, hidden and output layers), the total number of neurons indicating the melanoma and nevus classes may be computed as follows. In the input layer, 2,352 neurons are generated from the input being characterized by our $28 x 28 x 3$ color image vis-à-vis an output featuring a $2 x 1$ vector 


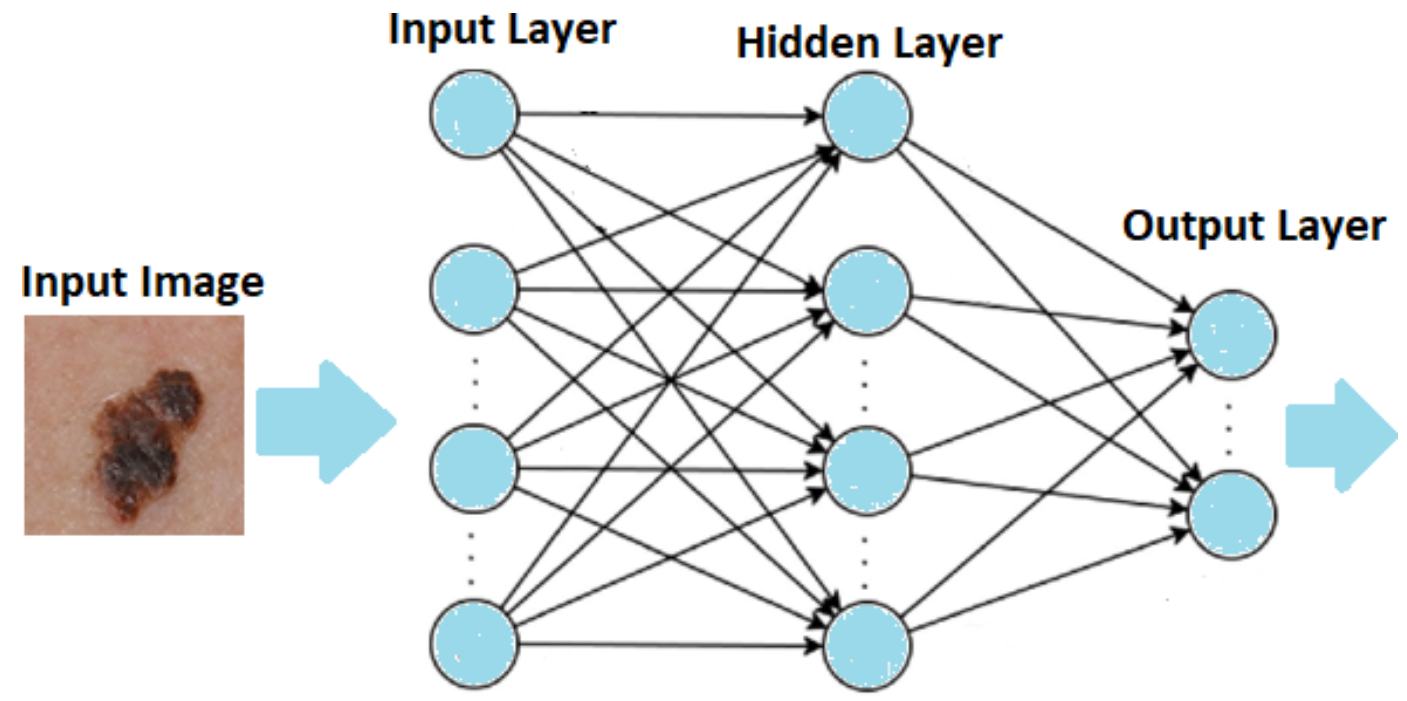

(or, two neurons). 500 neurons in the hidden layer are considered here. So, finally the MLP architecture comprises $2352+500+2=2854$ neurons. Importantly, an efficient variant of the gradient descent algorithm referred to as Adaptive Moment Estimation (Adam) is used as an optimization algorithm to service the complexity of this computation. Adam is preferred since it requires less memory and typically performs fine even with a minute tuning of hyperparameters.

\subsection{Convolution Neural Network (ConvNet)}

Convolutional neural network (ConvNet) is a deep learning algorithm, which has been applied by a growing number of researchers to various computer vision applications, resulting in a noticeable performance for equally complex problems. Importantly, ConvNet requires a much lower preprocessing power than other classification procedures (LeCun and Bengio, 1995). In contrast to the customary machine learning approaches such as Support Vector Machine (SVM) and traditional NN where handcrafted features are required to be extracted manually, deep learning models such as ConvNet utilize the raw input images to acquire and define features.

As characteristics of ANN, ConvNet also has an input, output, and one or multiple hidden layer(s) sandwiched in between the input-output layers. However, depending on the requirement of a particular application and the complexity of the problem task at hand, the topology of these networks often differs in the type and number of layers. As neurons in each ConvNet layer are organized in a threedimensional (3-D) structure, and transform into a 3-D output from a 3-D input, ConvNet is therefore particularly well suited for handling grid-like data, including data comprising 1-D information as in the case of signals such as audio, those comprising 2-D information as in the case of images, and those comprising 3-D information as in the case of videos. Accordingly, the ConvNet model applied in this skin lesion classification work has four convolutional layers as shown in Figure 2.

According to Chen et al. (2018), ConvNet classically has four layers: a convolutional layer, an activation layer, a pooling layer, and a dense layer as detailed below:

- Convolutional Layer: As the primary building block of a ConvNet, this layer essentially merges two sets of information. The word convolution denotes combining two functions via a mathematical operation to yield a third function. Hence, the key role of the convolution operation is to extract high-level features (for example, boundaries and edges in the input image). At the 


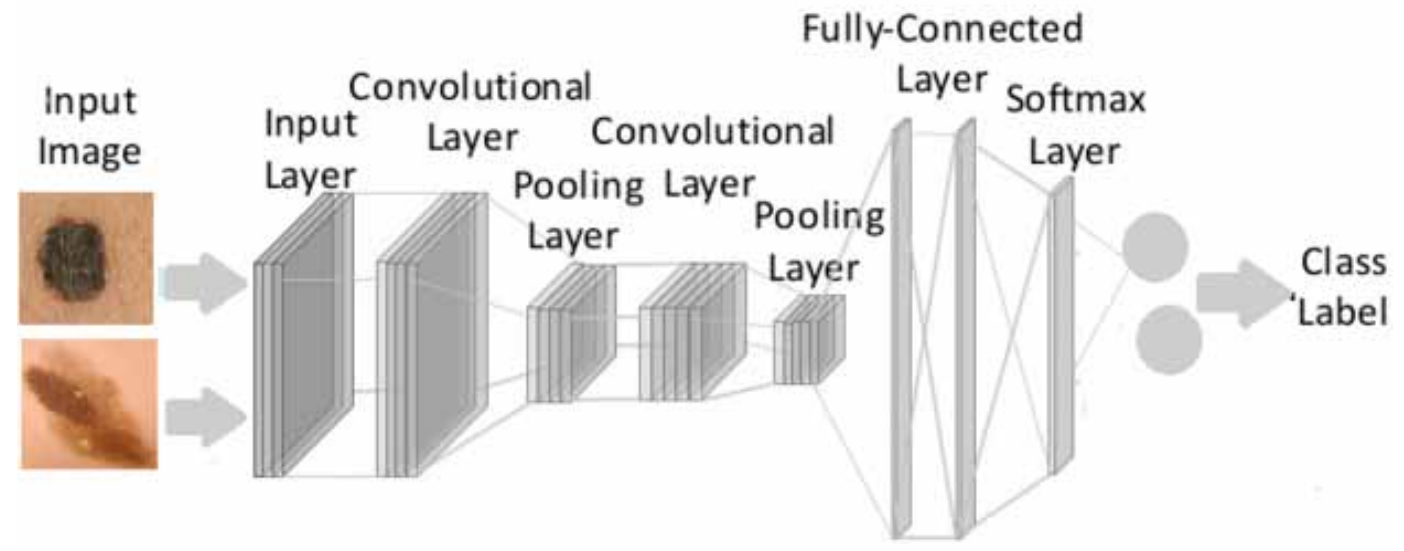

time of the forward pass, the convolution kernel slides across the dimensions of the image, thereby producing the representation of that region referred to as an activation map, which in turn provides the response of the kernel at each spatial location of the image. The sliding extent of the kernel is termed a stride.

- Activation Layer: Following each convolution layer, the next (activation) layer is required for accommodating nonlinearity. By permitting nonlinearity to be computed within a network that has just been performing linear operations via its convolution layers, the activation layer allows nonlinear functions such as sigmoid and tanh to be used in the neural network. Importantly, it has been shown that the default $\operatorname{ReLU}$ (rectified linear unit) activation function will often yield superior performance because for deep learning $\mathrm{NN}$, this function overcomes the vanishing gradient problem, allowing the network to train a lot faster without showing a significant difference to the accuracy. More simply, the $R e L U$ layer has the mapping function $f(x)=\max (0, x)$ to the input values and the layer just maps all the negative activations to 0 .

- Pooling Layer: A pooling layer is required to be added in between ConvNet layers after a convolution layer and activation layer. The aim here is dimensional reduction so as to lessen the number of training parameters and computational load onto the ANN. In other words, having this layer will ultimately reduce the learning time and also controls the overfitting of the models, thereby reducing the complexity of the computation. Amongst the various pooling operations, max pooling is a widely used operation that finds the maximum value in each window of a specified size.

- Fully Connected Layer: The fully connected layer (or dense layer) is generally used at the end of the ConvNet for classification purpose. It is just a regular layer of neurons within the ANN. It is thus densely connected as all neurons accept input from all the neurons of the preceding layer. Unlike the pooling and convolution layers, this layer entails a global operation as it receives input from the preceding layer and analyses the output of all preceding layers globally. Moreover, it creates a non-linear aggregation of certain features that are utilized for the classification of data (Guan et al., 2017).

As illustrated in Figure 2, the ConvNet model depicted here have the following sizes of layers for the first, second, third and fourth layer respectively: $28-3 x 3,64-3 x 3,128-3 x 3$ and $256-3 x 3$ convolution kernels. The nonlinear ReLU layers and max-pooling layers follow all of these convolution layers. All of the pooling windows of maximum operation have a window size of $2 x 2$. 
Altogether, the ANN has a fully connected layer of 50 neurons, and a final scoring layer with 2 neurons. This output layer comprises a softmax activation function (also called softargmax or normalized exponential function) with 2 .units corresponding to two classes, that is, melanoma vs. nevus. Softmax function yields a vector that signifies the probability distributions of a list of possible outcomes. The Adam optimizer, one of the widely acceptable optimization procedures, is used to compile the ConvNet model. The loss function used is given by binary cross-entropy and an inverse decay learning rate policy during training.

Deep NNs are considered very powerful with the high number of trainable parameters. Notwithstanding, in these networks, over-fitting can always become a severe issue. Moreover, these bulky sized networks are often also slow to apply. It is challenging to handle the overfitting just by uniting the predictions of multiple separate large neural nets during testing. A regularization mechanism known as "dropout" is a method for handling the overfitting issue. The basic principle here is to randomly drop some units from the ANN during the learning. A dropout layer with $0.2,0.3,0.3$ and 0.5 rates is used after first, second, third and last convolution layers respectively. To properly train a ConvNet model, one needs to decide a set of hyper-parameters, among which the learning rate is the most important one affecting the training performance. Here, it is set to 0.001 as decided via trials.

\subsection{Capsule Network (CapsNet)}

In a seminal paper, Hinton, et al., (2011) discuss on transforming auto-encoders, CapsNet. However, Sabour et al. (2017) offer the first CapsNet application in a work called Dynamic Routing between Capsules, where a CapsNet architecture is advocated as it reaches the state-of-the-art classification on Modified National Institute of Standards and Technology (MNIST) character image dataset even significantly better accuracy than ConvNet. Moreover, CapsNet also reduces some theoretical shortcomings of ConvNet, including:

- Pooling operations such as max-pooling operation, which drops the information about the spatial locations of the object in the scene; and

- ConvNet does not consider available spatial hierarchies between simpler and complex objects.

Hence, in preserving the hierarchical pose relationships between object parts with the intention of perfect classification and object identification, the application of CapsNet appears to be beneficial. The underlying rationale here, then, is to include relative relations between objects and symbolized numerically as a four-dimensional pose matrix (Xi et al., 2017) for applying CapsNet.

Capsule is a vector representing an element with size and direction. It is used to specify the features of the object and its likelihood. These features may be any of the instantiation parameters such as position, orientation, size, velocity, deformation, light reflection, texture, hue, and more (Afshar et al., 2018). As an alternative to simply capturing a feature with a particular variant, a capsule is learned to capture the similarities of a feature and other possible variants. Briefly, a capsule not only aims to identify a feature, but will also have the ability to train the model with variant features (Iesmantas et al., 2018). This makes CapsNet proficient for recognition based on inconsistencies in orientation and size of the object.

There are multiple layers of capsules in CapsNet. As shown in Figure 3, the proposed CapsNet architecture comprises a single ReLU convolution layer, followed by a layer of primary capsules (squashed and resized output of the convolutional layer), and a layer of SkinCaps (i.e. capsules signifying two categories of images: melanoma and nevus).

The convolution layer transforms pixel intensities to the activities of local feature finders, which are further passed as input to the primary capsules. In contrast to max-pooling layers of ConvNet, the convolution layer with strides larger than 2 is used for dimension reduction. The output of SkinCaps is utilized to decide the class of the input image. The entire capsules of the SkinCaps layer have a connection with every capsule in the primary capsule layer. Routing-by-agreement is a method that 


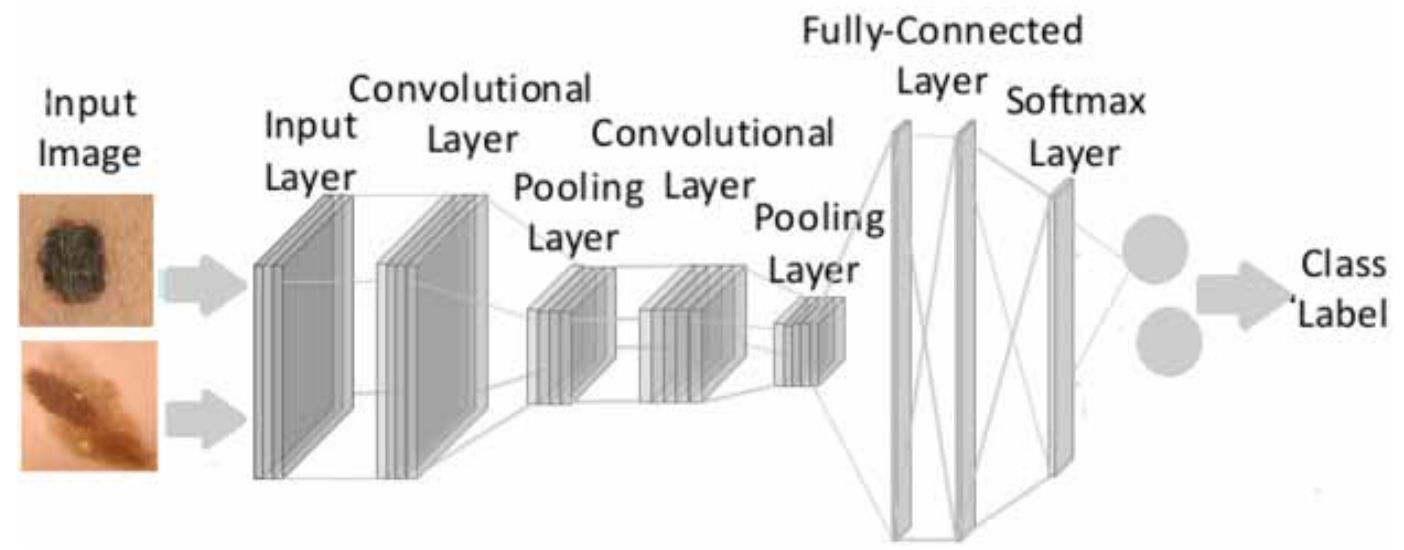

enables better learning in comparison to the max-pooling operation based routing. In the case of dynamic routing by agreement method, a feedback procedure that raises the support of those capsules that reach to the agreement most with the parent output, thereby further solidifying its support.

Notably, such activation functions in deep networks are used to approximate non-linear relationships by basic mathematical functions. These functions usually applied to scalar values. Notwithstanding, a special form of nonlinear activation function termed as a squash function is used in Capsule Networks to normalize the magnitude of the vectors.

This squash function is defined as:

$v_{j}=\frac{s_{j}^{2}}{1+s_{j}^{2}} \frac{s_{j}}{s_{j}}$

where $S_{j}$ is the weighted sum output of capsules given by:

$s_{j}=\sum_{i} c_{i j} \hat{u}_{j \mid i}$

and $\hat{u}_{j \mid i}$ represents the affine transformation defined as:

$\hat{u}_{j \mid i}=W_{i j} u_{i}$

\section{EXPERIMENTS}

In this section, we will offer details on the image dataset, the performance metrics and the different experiments together with their results vis-à-vis the application of all three notable classification models to be evaluated. 
Routing $\left(\hat{u}_{j \mid i}, x, y\right)$

Step 1: for all capsule node $i$ in layer $y$ and capsule node $j$ in layer $(y+1):$ bij $\leftarrow 0$

Step 2: for $x$ iterations do

Step 3: for all capsule node $i$ in layer $y: c_{i} \leftarrow \operatorname{softmax}\left(b_{i}\right)$

Step 4: for all capsule node $j$ in layer $(y+1): s_{j} \leftarrow \sum_{i} c_{i j} \hat{u}_{j \mid i}$

Step 5: for all capsule node $j$ in layer $(y+1): v_{j} \leftarrow \operatorname{squash}\left(s_{j}\right)$

Step 6: for all capsule node $i$ in layer $y$ and capsule node $j$ in layer $(y+1): b i j \longleftarrow b i j+\hat{u}_{j \mid i} \cdot v_{j}$ return $v_{j}$

\subsection{Dataset}

All of the three classification models are assessed on a publically offered image dataset of skin lesion images (Giotis et al., 2015).

The image dataset has non-dermoscopic images from the digital image collection of the dermatology department at the University Medical Center Groningen (UMCG). The total number of skin images in the dataset comprises 170 images, representing two classes of images, including (a) melanoma (70 samples); and (b) nevus (100 samples).

Owing to the limited size of image dataset, which may not be enough for training of the different models, an attempt is intentionally made to enlarge the dataset of one hundred seventy (170) images to two thousand and forty $(2,040)$ original and synthesized images by considering the rotated version of original images with angle 30 degree incrementally. Figure 4 provides sample images from the database for both of these two classes.

\subsection{Performance Metrics}

Four statistical performance metrics comprising: (a) precision, (b) recall, (c) F1-score, and (d) accuracy are computed from the confusion matrix to evaluate the different classification models. These metrics are defined as follows (Tiwari et al., 2017):

Figure 4. (a-b) Melanoma and nevus sample images
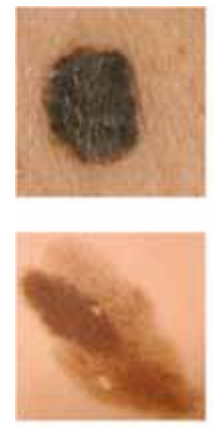
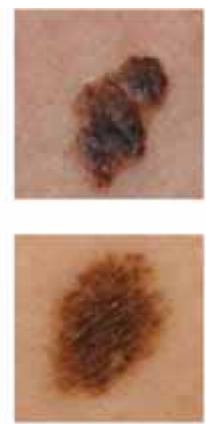
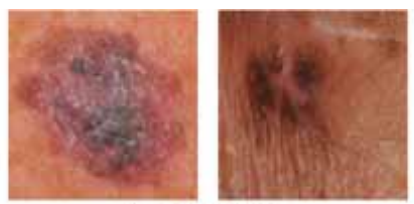

(a) melanoma

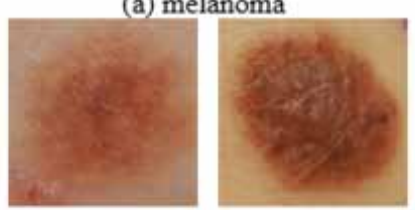

(b) nevus
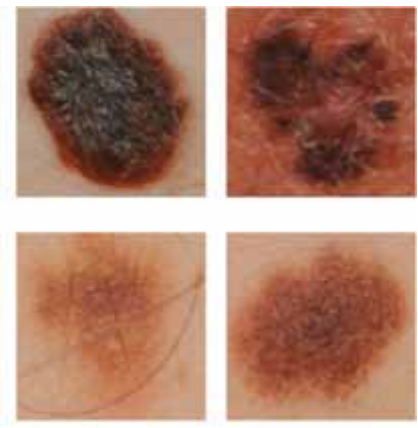
- Precision: Precision is the ratio of acceptably predicted positive cases to the total projected positive cases.

- Recall: Recall is the ratio of correctly projected positive cases to all cases in the actual classes:

$$
\text { Recall }=T^{+} /\left(T^{+}+F^{-}\right)
$$

- F1-Score: This score is the weighted average of Precision and Recall; therefore, this measure takes into consideration both false positives and false negatives into the calculation:

$$
F 1-\text { Score }=\left(2^{*} \text { Precision }^{*} \text { Recall }\right) /(\text { Precision }+ \text { Recall })
$$

- Accuracy: Accuracy, a ratio of acceptably projected cases to the total cases, is the most native performance metric:

$$
\text { Accuracy }=\left(T^{+}+T^{-}\right) /\left(T^{+}+T^{-}+F^{-}+F^{+}\right)
$$

where:

- $\quad \mathrm{T}^{+}$(True Positive) and $\mathrm{T}^{-}$(True Negative) is the truly projected positive and negative cases respectively.

- $\quad \mathrm{F}^{+}$(False Positive) indicates the inaccurately projected positive cases.

- $\mathrm{F}^{-}$(False Negative) indicates the inaccurately projected negative cases.

To further assess the robustness of the models, the learning curves for both the training and validation data are plotted. From these learning curves, overfitting and underfitting of the model may be more easily detected and recognized. For example, if these curves are close to each other and both of them but do not have a high score, then the model has under-fitted, implying a high biased.

Conversely, if the training curve has a higher score, but the validation curve has a lower score, that is, there are big gaps between these curves, then the model is likely over-fitted or there will likely to be a high variance.

\subsection{Experiment 1: MLP for Melanoma and Nevus Classification}

As discussed in Section 3, MLP model is used for melanoma and nevus categorization in this experiment. One hundred (100) epochs are used for training of the model with a division of $70 \%$ and $30 \%$ being carried out between the training and validation data. This implies that $70 \%$ of the dataset will be used to train the MLP model, whereas the remaining $30 \%$ of the dataset will be used to validate the trained model.

All the skin images are utilized for testing in this experiment. The trained model has reached to maximum accuracy of $76.4 \%$. Figure 5 displays the accuracy and loss curves throughout learning with the training epochs set to 100 . 
Figure 5. (a) Accuracy curves for MLP model (b) Loss curves for experiment with MLP model

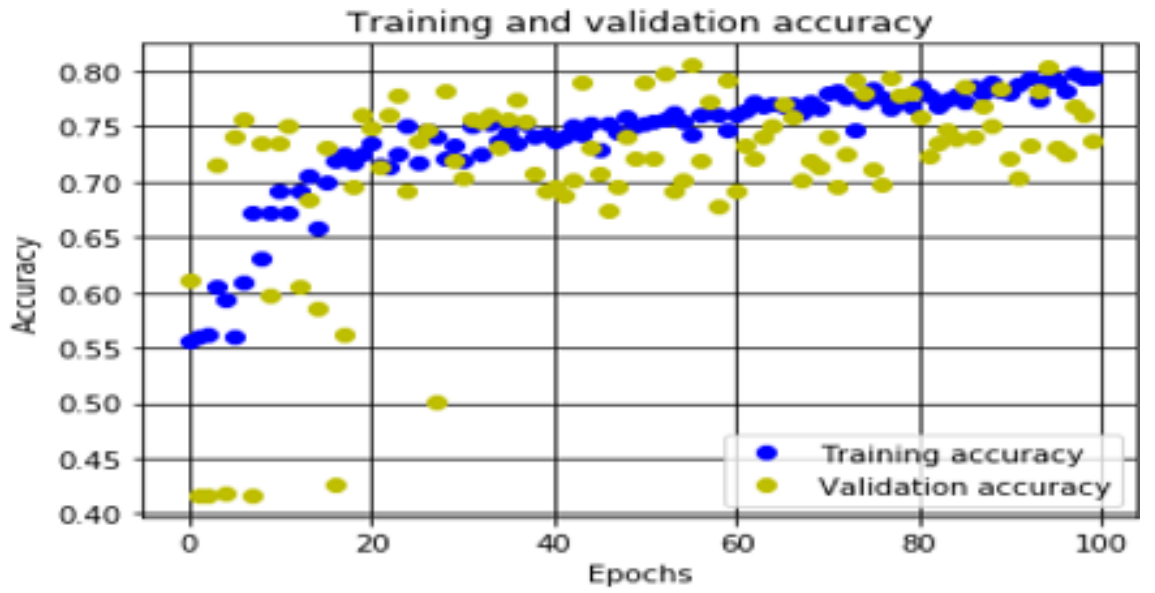

(a)

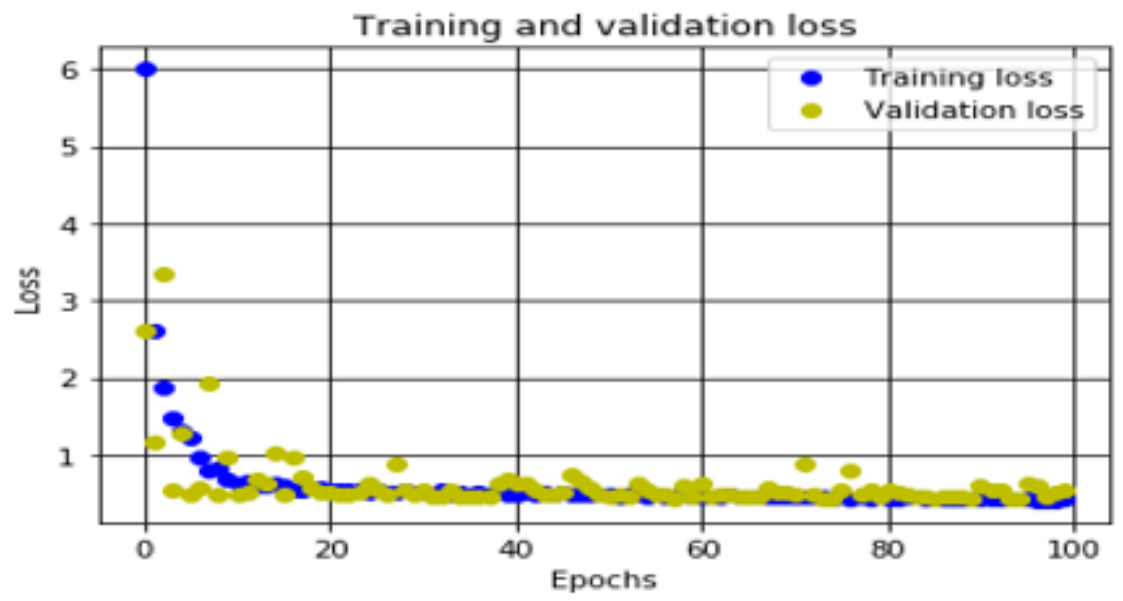

(b)

During training, the training loss continues to decrease while training accuracy continues to increase until convergence. Even though these curves are non-linear and closed to each other, it still demonstrates that the MLP model has not achieved high accuracy.

The performance metrics computed separately for both classes are given in Table 1 .

\subsection{Experiment 2: ConvNet for Melanoma and Nevus Classification}

Again, as noted in Section 3, the ConvNet model will be evaluated for melanoma and nevus categorization in this next experiment.

One hundred (100) epochs are found suitable for the training of the ConvNet model with a division of $70 \%$ and $30 \%$ being split between the training and validation data. This means that $70 \%$ of the data is used to train the ConvNet, whereas $30 \%$ of the data is used to validate the trained ConvNet. Once again, all the skin images are utilized for testing in this experiment. The accuracy attained from the trained ConvNet model is $95.1 \%$. Figure 6 shows these curves throughout learning with total training epochs set to 100 . During training, the training loss continues to decrease whereas training accuracy continues to increase until convergence. Even though the validation loss and accuracy 
Table 1. Performance measures with MLP

\begin{tabular}{|l|l|l|l|}
\hline \multicolumn{1}{|c|}{ Class } & \multicolumn{1}{c|}{ Precision } & Recall & f1-Score \\
\hline Melanoma & 0.84 & 0.53 & 0.65 \\
\hline Nevus & 0.74 & 0.93 & 0.82 \\
\hline Average & 0.78 & 0.76 & 0.75 \\
\hline
\end{tabular}

Figure 6. (a) Accuracy curves for experiment with CNN model (b) Loss curves for experiment with CNN model

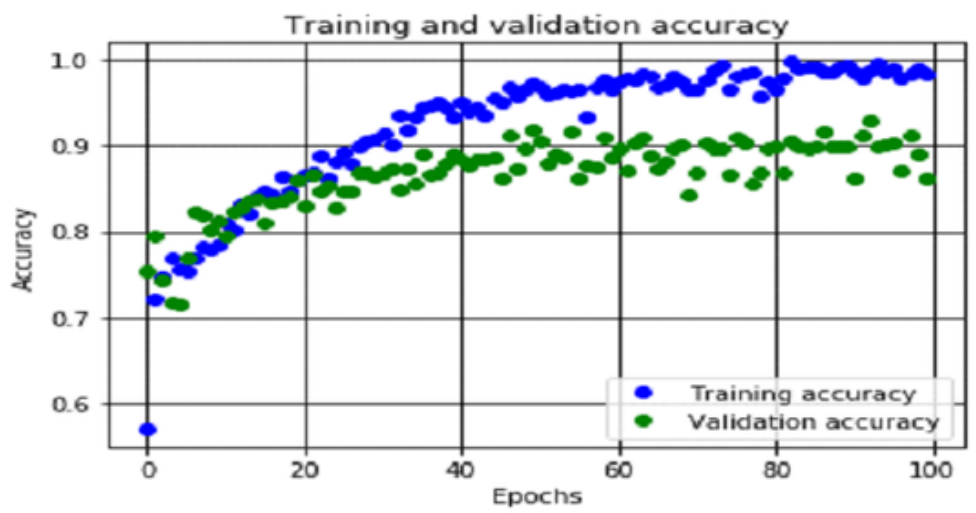

(a)

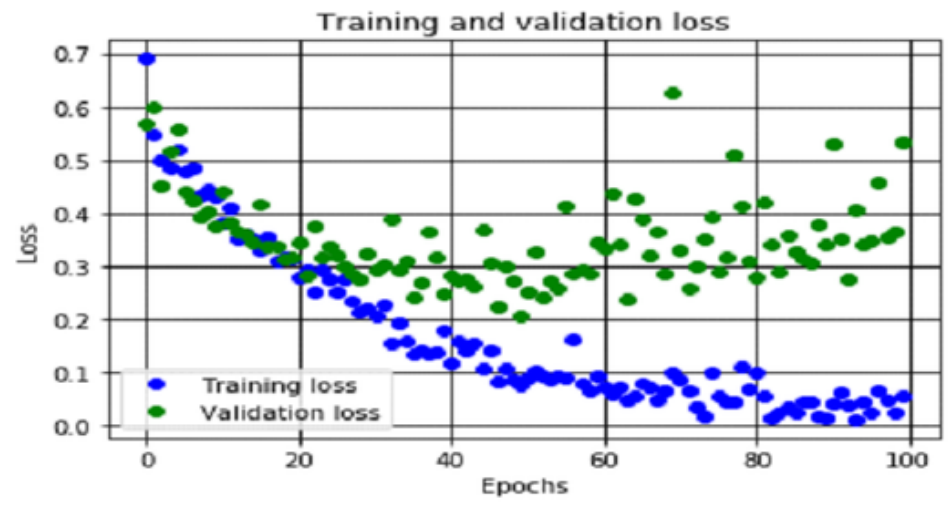

(b)

curves are non-linear and slightly apart from each other, these resulting curves still demonstrate that the ConvNet model is achieving good accuracy with reasonable control of over- and under-fitting the model.

The performance metrics are detailed in Table 2 for both the classes.

\subsection{Experiment 3: CapsNet for Melanoma and Nevus Classification}

As noted in Section 3, the CapsNet model is used for melanoma and nevus categorization in this final experiment. Once again, the model is trained over 100 epochs with a division of $70 \%$ and $30 \%$ between the training and validation data. This implies that $70 \%$ of the data is used to train the CapsNet model, and $30 \%$ of the data is used to validate the trained model. 
Table 2. Performance measures with CNN

\begin{tabular}{|l|l|l|l|}
\hline \multicolumn{1}{|c|}{ Class } & \multicolumn{1}{c|}{ Precision } & \multicolumn{1}{c|}{ Recall } & \multicolumn{1}{c|}{ f1-Score } \\
\hline Melanoma & 0.99 & 0.89 & 0.94 \\
\hline Nevus & 0.93 & 0.99 & 0.96 \\
\hline Average & 0.95 & 0.95 & 0.95 \\
\hline
\end{tabular}

Just as with the other experiments, all of the available images are used for this evaluation. The trained model has reached an accuracy of $98.9 \%$. Figure 7 displays the accuracy and loss curves throughout learning with the maximum training epochs set to 100 .

As depicted by the resulting curves, during training, the training loss is shown to keep decreasing while training accuracy keeps increasing until convergence. Moreover, even though these curves are nonlinear and slightly apart from each other, results still demonstrate that the trained CapsNet model is well generalized and portrayed adequate accuracy for both training and validation data.

Performance metrics are presented in Table 3.

Finally, in the result analysis subsection, we compare the evaluation results.

Figure 7. (a) Accuracy curves of for experiment with Capsule network model (b) Loss curves for experiment with Capsule network model

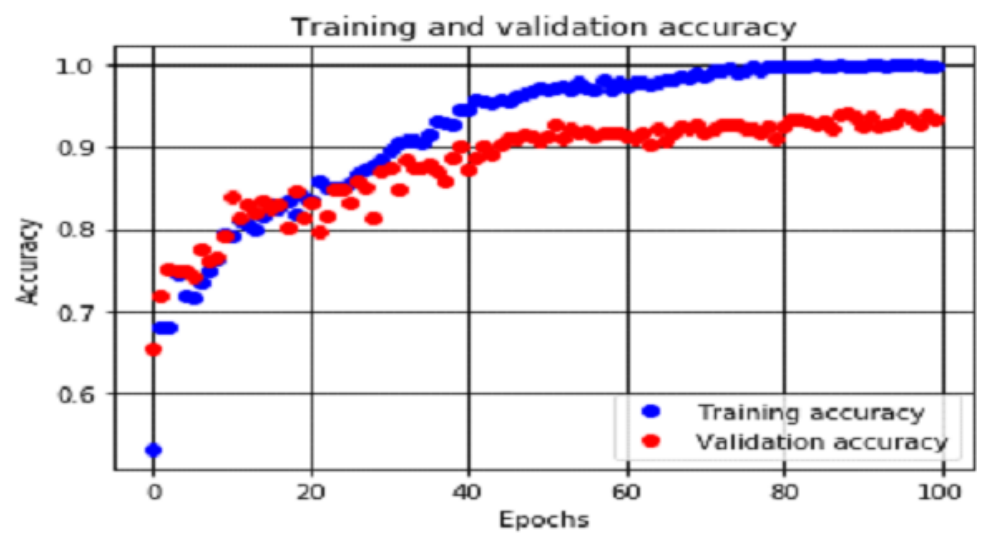

(a)

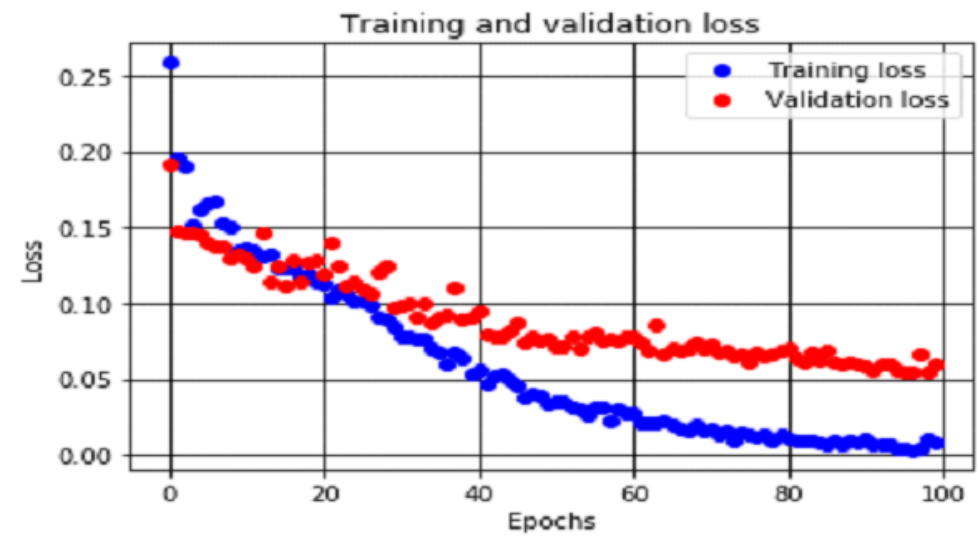

(b) 
Table 3. Performance measures with Capsule Network

\begin{tabular}{|l|l|l|l|}
\hline \multicolumn{1}{|c|}{ Class } & \multicolumn{1}{c|}{ Precision } & Recall & f1-Score \\
\hline Melanoma & 0.96 & 0.98 & 0.97 \\
\hline Nevus & 0.99 & 0.97 & 0.98 \\
\hline Average & 0.98 & 0.98 & 0.98 \\
\hline
\end{tabular}

\subsection{Result Analysis}

The average precision metrics are found as $0.78,0.95$, and 0.98 respectively for MLP, CNN and CapsNet models. The recall values are found 0.76, 0.95, and 0.98 respectively for MLP, CNN and CapsNet models. The third performance metric i.e. f1-score is calculated and 0.78, 0.95, and 0.98 values are found correspondingly for MLP, CNN and CapsNet models. It is evident that CapsNet model performs better than other two models. Based on the additional analysis, the average accuracies achieved among the three different models being evaluated, that is, MLP, ConvNet, and CapsNet, are respectively increasing to $76.4 \%, 95.1 \%$ and $98.9 \%$. Apparently, the performance of the CapsNet model is superior to the other two models. To summarize the results, the graphs of validation accuracies are also plotted in Figure 8. These plots also confirm that the Capsule network outperforms than MLP and ConvNet.

\section{CONCLUSION}

This study reported on the application of three different classification models namely multi-layer perceptron, convolution neural network and capsule network to distinguish malignant melanoma from benign nevus. It is confirmed from the simulation results that the capsule network is the most suitable classification model for these images.

Figure 8. Validation accuracies plots for experiment with all three models

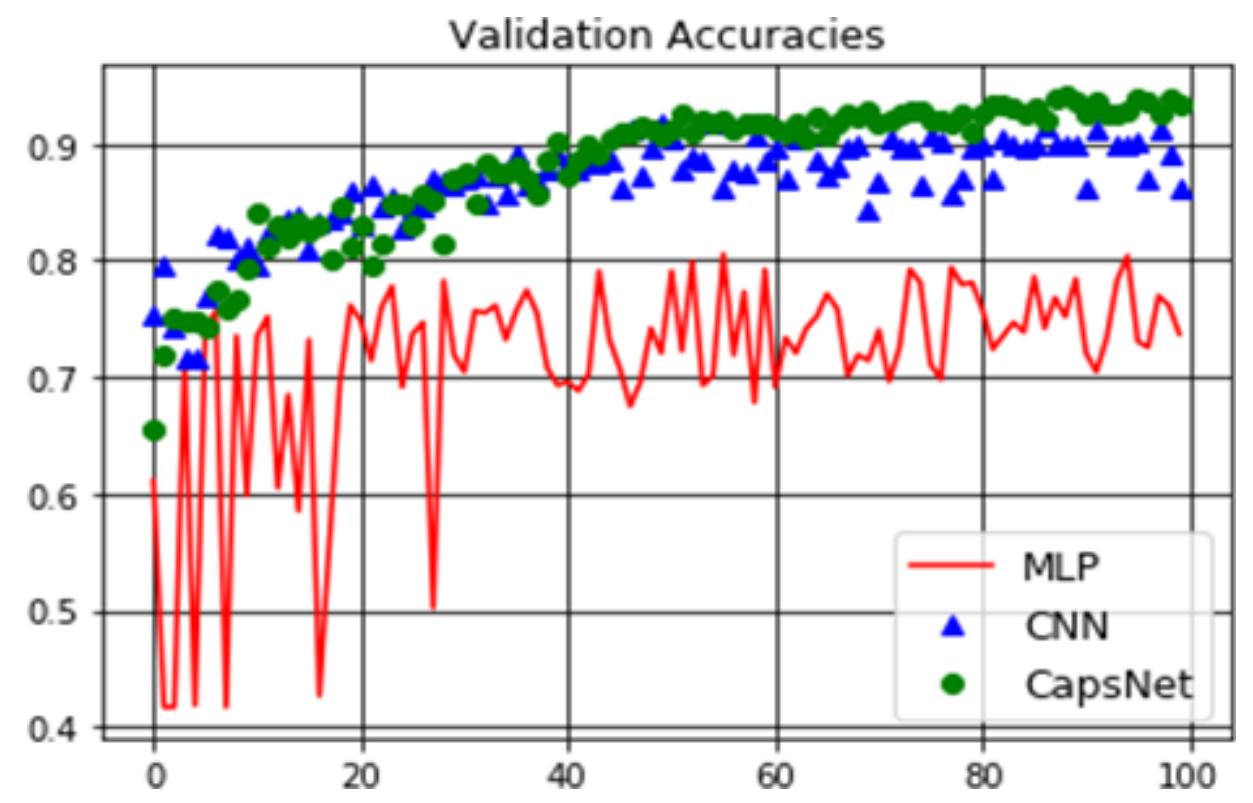


Capsule network yields best accuracy than other models. Capsule network utilizes capsules that group neurons and in this manner decreases the number of connections necessary as compared to a convolution neural network. It is demonstrated that a CapsNet model with fewer learning parameters can be generalized in a better manner and attains better performance for skin cancer detection. Therefore, a capsule network model can be preferred over other classification models.

Though the capsule networks are definitely performing better for skin cancer detection than multi layer perceptron and convolutional neural networks, there is more scope is available to attain better accuracies with improved network architectures, and superior capsule and routing procedures. Moreover, the solutions based on these deep learning architectures always have some technical limitations such as requirement of large dataset, high-performance computational resources and extensive learning times.

This work further explores the possibilities of an improvement in classification accuracies by adding clinical data for example age, gender, race, skin type, and other demographic information as inputs for the multi channel classifiers. This supplementary information is valuable for the classification. Future work should consider these characteristics into account. 


\section{REFERENCES}

Afshar, P., Mohammadi, A., \& Plataniotis, K. N. (2018). Brain tumor type classification via capsule networks. 10.1109/ICIP.2018.8451379

Alcón, J. F., Ciuhu, C., Ten Kate, W., Heinrich, A., Uzunbajakava, N., Krekels, G., \& De Haan, G. et al. (2009). Automatic imaging system with decision support for inspection of pigmented skin lesions and melanoma diagnosis. IEEE Journal of Selected Topics in Signal Processing, 3(1), 14-25. doi:10.1109/JSTSP.2008.2011156

Argenziano, G., Cerroni, L., Zalaudek, I., Staibano, S., Hofmann-Wellenhof, R., Arpaia, N., \& Brunasso, A. M. (2012). Accuracy in melanoma detection: A 10-year multicenter survey. Journal of the American Academy of Dermatology, 67(1), 54-59. doi:10.1016/j.jaad.2011.07.019 PMID:21982636

Argenziano, G., Fabbrocini, G., Carli, P., De Giorgi, V., Sammarco, E., \& Delfino, M. (1998). Epiluminescence microscopy for the diagnosis of doubtful melanocytic skin lesions: Comparison of the ABCD rule of dermatoscopy and a new 7-point checklist based on pattern analysis. Archives of Dermatology, 134(12), 1563-1570. doi:10.1001/ archderm.134.12.1563 PMID:9875194

Carcagnì, P., Cuna, A., \& Distante, C. (2018). A Dense CNN approach for skin lesion classification. arXiv preprint arXiv:1807.06416

Cavalcanti, P. G., \& Scharcanski, J. (2011). Automated prescreening of pigmented skin lesions using standard cameras. Computerized Medical Imaging and Graphics, 35(6), 481-491. doi:10.1016/j.compmedimag.2011.02.007 PMID:21489751

Chen, L. C., Papandreou, G., Kokkinos, I., Murphy, K., \& Yuille, A. L. (2018). Deeplab: Semantic image segmentation with deep convolutional nets, atrous convolution, and fully connected crfs. IEEE Transactions on Pattern Analysis and Machine Intelligence, 40(4), 834-848. doi:10.1109/TPAMI.2017.2699184 PMID:28463186

Esteves, J. T., de Souza Rolim, G., \& Ferraudo, A. S. (2018). Rainfall prediction methodology with binary multilayer perceptron neural networks. Climate Dynamics, 1-13.

Giotis, I., Molders, N., Land, S., Biehl, M., Jonkman, M. F., \& Petkov, N. (2015). MED-NODE: A computerassisted melanoma diagnosis system using non-dermoscopic images. Expert Systems with Applications, 42(19), 6578-6585. doi:10.1016/j.eswa.2015.04.034

Guan, G., Zhang, F., Ding, G., Niu, M., \& Xu, L. (2017). A Video Colouring Method Based on CNN and Feature Point Tracking. In International Conference on Smart Vehicular Technology, Transportation, Communication and Applications (pp. 312-320). Springer.

Hinton, G. E., Krizhevsky, A., \& Wang, S. D. (2011, June). Transforming auto-encoders. In International Conference on Artificial Neural Networks (pp. 44-51). Springer.

Iesmantas, T., \& Alzbutas, R. (2018). Convolutional capsule network for classification of breast cancer histology images. In International Conference Image Analysis and Recognition (pp. 853-860). Springer. doi:10.1007/9783-319-93000-8_97

Ker, J., Wang, L., Rao, J., \& Lim, T. (2018). Deep learning applications in medical image analysis. IEEE Access: Practical Innovations, Open Solutions, 6, 9375-9389. doi:10.1109/ACCESS.2017.2788044

Kruse, R., Borgelt, C., Klawonn, F., Moewes, C., Steinbrecher, M., \& Held, P. (2013). Multi-layer perceptrons. In Computational Intelligence (pp. 47-81). Springer.

LeCun, Y., \& Bengio, Y. (1995). Convolutional networks for images, speech, and time series. The handbook of brain theory and neural networks, 3361(10).

Mukherjee, S., Adhikari, A., \& Roy, M. (2018). Melanoma Identification Using MLP With Parameter Selected by Metaheuristic Algorithms. Intelligent Innovations in Multimedia Data Engineering and Management, 241.

Nanni, L., Lumini, A., \& Ghidoni, S. (2018). Ensemble of Deep Learned Features for Melanoma Classification. arXiv preprint arXiv: 1807.08008

Rigel, D. S., Russak, J., \& Friedman, R. (2010). The evolution of melanoma diagnosis: 25 years beyond the ABCDs. CA: a Cancer Journal for Clinicians, 60(5), 301-316. PMID:20671054 
Sabour, S., Frosst, N., \& Hinton, G. E. (2017). Dynamic routing between capsules. In Advances in Neural Information Processing Systems (pp. 3856-3866). Academic Press.

Sheha, M. A., Mabrouk, M. S., \& Sharawy, A. (2012). Automatic detection of melanoma skin cancer using texture analysis. International Journal of Computers and Applications, 42(20), 22-26.

Singhal, E., \& Tiwari, S. (2015). Skin Cancer Detection using Arificial Neural Network. International Journal of Advanced Research in Computer Science, 6(1).

Tiwari, S. (2017). A Pattern Classification Based approach for Blur Classification. Indonesian Journal of Electrical Engineering and Informatics, 5(2), 162-173.

Tiwari, S., Singh, A. K., \& Shukla, V. P. (2011). Statistical moments based noise classification using feed forward back propagation neural network. International Journal of Computers and Applications, 18(2), 36-40.

Xi, E., Bing, S., \& Jin, Y. (2017). Capsule network performance on complex data. arXiv preprint arXiv:1712.03480.

Yu, L., Chen, H., Dou, Q., Qin, J., \& Heng, P. A. (2017). Automated melanoma recognition in dermoscopy images via very deep residual networks. IEEE Transactions on Medical Imaging, 36(4), 994-1004. PMID:28026754 